\title{
Plaque lesion type chromoblastomycosis caused by Fonsecaea monophora: Case report
}

\author{
Rodrigo Vettorato1,2; Daiane Heidrich1; Danielle Machado Pagani3; Amanda Carvalho \\ Ribeiro2; Gerson Vettorato2; Taís Guarienti Amaro2; Maria Lucia Scroferneker1,4
}

1 Postgraduate Program in Medicine: Medical Sciences, Universidade Federal do Rio Grande do Sul. Rua Ramiro Barcelos, 2400 - $2^{\text {nd }}$ floor, Porto Alegre, CEP: 90035-003, Brazil.

2 Dermatology Service of Complexo Hospitalar Santa Casa de Misericórdia de Porto Alegre. Rua Professor Annes Dias - 135, Porto Alegre, CEP: 90020-090, Brazil.

3 Postgraduate Program in Agricultural and Environmental Microbiology, Universidade Federal do Rio Grande do Sul.

Rua Sarmento Leite, 500 - 210, Porto Alegre, CEP: 90050170, Brazil.

4 Department of Microbiology, ICBS, Universidade Federal do Rio Grande do Sul. Rua Sarmento Leite, 500, Porto

Alegre, CEP: 90050-170, Brazil.

Objectives: to report a case of chromoblastomycosis with plaque lesion type, the least common type lesion of the disease, that was caused by Fonsecaea monophora in Rio Grande do Sul, Brazil, and, additionally, antifungal assay was performed.

Methods: the lesion on arm of a 65-years-old man had slow and progressive desenvolver during five years. On physical examination, erythematous plaque, formed by the coalescence of multiple papules, with well defined borders and discreet surface scaling (Figure 1). In dermatoscopy, there was erythema with vascular polymorphism (irregular, glomerular and dotted linear vessels), and multiple bright white lines, acquired orthogonal arrangement in some regions, delimiting areas with the presence of red-orange points. Against these characteristics, initial hypotheses were cutaneous nonmelanoma skin cancer, cutaneous lymphoma or sarcoidosis or atypical mycobacteriosis (tiraria micobacteriose). For diagnostic, an incisional biopsy with punch $4 \mathrm{~mm}$ was realized. The stains hematoxilin and eosin, Zhiel Neelsen and Groccot were performed. Besides that, mycological cultural examination in Mycosel medium was requested after fungal evidence. For species identification, the sequencing of Internal Transcribed Spacer (ITS) of rDNA using primers ITS 1 and 4 was performed comparing the sequence with the type strains of the genus in phylogenetic tree. The minimal inhibitory concentrations (MICs) of amphotericin B, itraconazole, ketoconazole, voriconazole, posaconazole and terbinafine (all Sigma-Aldrich, USA) were evaluating according to the protocol M38-A2 of the Clinical and Laboratory Standards Institute (CLSI), using the microdilution technique. The minimum fungicidal concentrations (MFCs) were also determined.

Results: anatomopathology showed pseudoepitheliomatous epithelial hyperplasia, granulomas in the superficial dermis with prominent peripheral lympho-histiocytic infiltrate and sclerotic cells, indicating chromoblastomycosis (Figure 2A,B). Besides, Ziehl Neelsen was negative and Grocott was positive. In culture, colony of Fonsecaea spp. (Figure 2C) was developed and the species, F. monophora, was identified by ITS sequencing.
The MICs obtained of antifungals were $(\mu \mathrm{g} / \mathrm{mL})$ : posaconazole $(\leq 0.03)$; ketoconazole and terbinafine $(0.06)$; itraconazole and voriconazole (0.125); amphotericin B (>16.0).

The MFC of terbinafine was $0.5 \mu \mathrm{g} / \mathrm{mL}$ and for all the others, the MFC were $\geq 4.0 \mu \mathrm{g} / \mathrm{mL}$.

Excepting amphotericin B, the isolate had lower MICs for all other antifungals, including itraconazole, which is the unique oral antifungal with free governmental distribution in Brazil. Therefore, itraconazole $400 \mathrm{mg} /$ day was prescribed for the patient, which is being accompanied by the medical staff and showed improvement of the lesion during six months of treatment.

Conclusions: chromoblastomycosis was not among the initial hypotheses of diagnosis, since the atypical lesion (plaque type) of this case. However, the pathology requested was determinant for the diagnosis of the disease. In addition, this report shows that $F$. monophora has been taking place in the diagnosis in the country with the highest prevalence of Fonsecaea pedrosoi.

Figure 1.

Chromoblastomycosis with plaque lesion caused by Fonsecaea monophora on the arm.

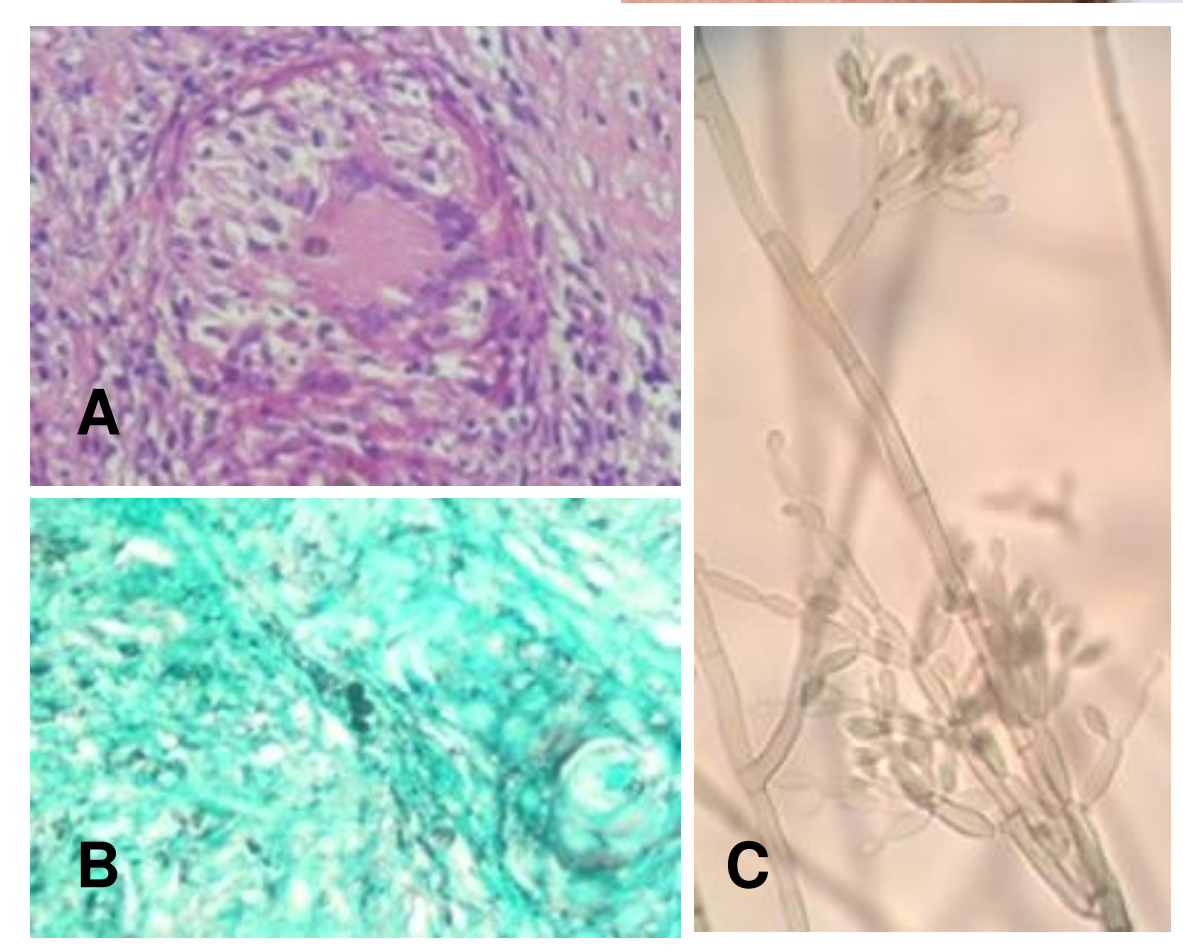

Figure 2. Anatomopathology with hematoxilin and eosin (A) and Grocott (B), indicating chromobastomycosis and the identification of Fonsecaea sp. by microcultive (C). 